\title{
Non-alcoholic fatty liver diseases: update on the challenge of diagnosis and treatment
}

\author{
Hyunwoo Oh', ${ }^{1,2}$ Dae Won Jun', Waqar K Saeed', and Mindie H Nguyen ${ }^{3}$ \\ Department of Internal Medicine, Hanyang University College of Medicine, ${ }^{2}$ Medical corp, 7th division, Republic of Korea army, \\ ${ }^{3}$ Division of Gastroenterology and Hepatology, Stanford University Medical Center, Palo Alto, CA, USA
}

The prevalence of non-alcoholic fatty liver disease (NAFLD) is estimated to be $25-30 \%$ of the population, and is the most common cause of elevated liver enzymes in Korea. NAFLD is a "hot potato" for pharmaceutical companies. Many clinical trials are underway to develop a first-in-class drug to treat NAFLD. However, there are several challenging issues regarding the diagnosis of NAFLD. Currently, liver biopsy is the gold standard method for the diagnosis of NAFLD and steatohepatitis. Ideally, globally recognized standards for histological diagnosis and methods to optimize observer agreement on biopsy interpretation should be developed. Liver biopsy is the best method rather than a perfect one. Recently, multi-parametric magnetic resonance imagery can estimate the amount of intrahepatic fat successfully and is widely used in clinical trials. But no diagnostic method can discriminate between steatohepatitis and simple steatosis. The other unresolved issue in regard to NAFLD is the absence of satisfactory treatment options. Vitamin E and obeticholic acid have shown protective effects in randomized controlled trials, but this drug has not been approved for use in Korea. This study will provide a description of diagnostic methods and treatments that are currently recommended for NAFLD. (Clin Mol Hepatol 2016;22:327-335)

Keywords: Non-alcoholic fatty liver; Diagnosis; Treatment

\section{INTRODUCTION}

Non-alcoholic fatty liver disease (NAFLD) is a disease complex that includes simple steatosis (fatty accumulation in the liver), non-alcoholic steatohepatitis (NASH) (inflammatory cell infiltration in the liver), hepatocyte ballooning, and hepatic cirrhosis associated with NAFLD. In Korea the prevalence of fatty liver may be as high as $25-30 \%,{ }^{1} 10-15 \%$ of whom may have steatohepatitis with inflammation. In clinical practice, NASH, including inflammation and fibrosis, is an important diagnosis, and sometimes leads to hepatic cirrhosis and liver cancer. Currently, NAFLD is the most common chronic liver disease in developed countries.
Unresolved issues relating to NAFLD are the absence of effective diagnostic methods and satisfactory treatments. This study will provide a description of diagnostic methods and treatments that are currently recommended for NAFLD.

\section{DIAGNOSIS OF NAFLD: NON-INVASIVE METHOD I (radiologic method)}

\section{Ultrasonography}

Abdominal ultrasonography (US) is one of the most widely used

\section{Corresponding author : Dae Won Jun}

Abbreviations:

AUROC, areas under the receiver-operating curve; CK-18, cytokeratin-18; $\mathrm{CT}$, computed tomography; $\mathrm{HU}$, Hounsfield unit; $F X R$, farnesoid X receptor: MRS, magnetic resonance spectroscopy; NAFLD, non-alcoholic fatty liver disease; NASH, non-alcoholic steatohepatitis; NFS, NAFLD Fibrosis Score: NPV, negative predictive value; PDFF, proton density fat fraction; PPV, positive predictive value; SAF, scoring system for steatosis, activity, and fibrosis

\section{Department of Internal Medicine, Hanyang University College of}

Tel: +82-2-2290-8338, Fax: +82-2-972-0068

E-mail: noshin@hanyang.ac.kr Medicine, 220 Wangsimni-ro, Seongdong-gu, Seoul 04763, Korea 
one of the fatty liver in clinical practice. It is relatively inexpensive and has a sensitivity of $93 \%$, high enough to diagnose a moderately fatty liver with more than $33 \%$ fat. $^{2}$ However, US shows low specificity and low sensitivity if the liver is composed of less than $30 \%$ fat. There also may be a difference in diagnosis due to variations in the interpretations of the readers of the ultrasonograms. ${ }^{2}$

\section{Computed tomography}

Abdominal computed tomography (CT) produces different radiolucency depending on the individual organ examined. The measurement of this radiolucency is called the Hounsfield unit (HU), named after an English electrical engineer who discovered this variation. In general, the calibration of images of body tissues is normalized based on values for air $(-1,000 \mathrm{HU})$, water $(0 \mathrm{HU})$, and bone density $(+1,000 \mathrm{HU})$. Unenhanced abdominal CT uses liver $\mathrm{HU}$ and spleen $\mathrm{HU}$ to determine the degree of steatosis. The liver, which has a value of $60 \pm 10 \mathrm{HU}$, normally looks brighter than the spleen, blood vessels or biliary tract, while subcutaneous fat represents a value of $-90 \mathrm{HU}^{3}$ Increased fat accumulation in the liver may lead to a liver HU that is lower than spleen HU. Generally, liver $\mathrm{HU} \leq 40 \mathrm{HU}$, or liver minus spleen density difference less than -10 HU, or liver/spleen ratio of $\leq 0.9$, which can lead to the diagnosis of hepatic steatosis. ${ }^{3}$ However, there are several disadvantages to this method. Radiation exposure, or HU, can differ depending on the set-up of $\mathrm{CT}$ machines by suppliers and installers, and HU can be affected by various conditions, such as accompanying edema or accumulation of copper in liver. CT is not superior to ultrasonography in the assessment of hepatic steatosis, but CT can suggest quantitative and objective values in comparison with ultrasonography.

\section{Multi-parametric magnetic resonance imaging (MRI) of quantitative fat}

Hydrogen in fat is different in resonance from hydrogen in water. Magnetic resonance imaging proton density fat fraction (MRIPDFF) has been widely used to estimate the level of hepatic triglycerides by calculating the fraction of triglycerides versus hydrogen in water. The sensitivity and specificity of MRI-PDFF using a multi-echo technique to estimate intrahepatic fat amount is nearly perfect. ${ }^{4,5}$ MRI-PDFF is known as a reference standard in the field of radiology to measure the amount of hepatic fat. ${ }^{6}$ Such MRI-based hepatic fat measurement has been used by a number of multinational corporations that produce MRI machines, such as
IDEAL IQ ${ }^{\circledR}(G E)$, mDixon Qant ${ }^{\circledR}$ (Philips), and Multiecho VIBE Dixon ${ }^{\circledR}$ (Siemens). Additionally, clinical use has been approved by the Federal Drug Administration (FDA) in the U.S., in which MRI-PDFF can be a substitute for liver biopsy in randomized controlled trials that use hepatic fat change as the primary variable. ${ }^{7.8}$ MRI-PDFF values are: $0-6.4 \%$ for grade 0 (normal) with less than $5 \%$ hepatic fat from tissue biopsy; $6.5-17.5 \%$ for grade 1 (mild) with 5-33\% hepatic fat; $17.5-22.1 \%$ for grade 2 (moderate) with $33-66 \%$ hepatic fat; and $22.2 \%$ or greater for grade 3 (severe) with more than $66 \%$ hepatic fat. ${ }^{9,10}$ Nonetheless, it is difficult for primary care and other facilities to use this high-cost equipment universally. But the most important limitation of MRI-PDFF is that it can estimate the intrahepatic fat amount almost perfectly, but cannot detect the difference between NASH and NAFLD.

\section{DIAGNOSIS OF NAFLD : NON-INVASIVE METHOD II (serologic method)}

There are three serologic methods used to diagnose NAFLD; the first, a test for predicting hepatic steatosis; the second, a method for determining NASH, and lastly, a test for detecting advanced fibrosis $\geq \mathrm{F} 3$.

\section{Serologic testing for simple steatosis}

The fatty liver index ${ }^{11}$ and SteatoTest ${ }^{12}$ have been developed to detect simple steatosis, but are not in widespread use due to the availability of advanced imaging tools, such as ultrasonography or MR imaging-PDFF.

\section{Serologic testing for NASH}

Although radiologic assessment of NASH has improved, no radiologic method can detect a difference between NASH and NAFLD. Many studies on serological surrogate markers are used to distinguish steatohepatitis from the inflammation of simple steatosis, and single serological test methods to predict steatohepatitis have also been studied. Although the recommended surrogate markers TNF- $\alpha$, IL-6, CRP, Pantraxin, Ferritin, SPEA, and SRAGE predict hepatic inflammation, most of these markers still need extensive external validation (Table 1)..$^{13-19}$ The single test most studied so far in regard to the diagnosis of NASH is Cytokeratin-18 (CK-18). The CK-18 fragment, a marker of hepatocyte apoptosis, predicts nonalcoholic steatohepatitis, which is signifi- 
Table 1. Single biomarkers of non-alcoholic steatohepatitis

\begin{tabular}{lcccc}
\hline & Cut off & AUROC & Sensitivity (\%) & Specificity (\%) \\
\hline TNF-a $^{13}$ & $100 \mathrm{ng} / \mathrm{mL}$ & 0.685 & 66.7 & 74.1 \\
\hline $\mathrm{IL}^{14}{ }^{14}$ & $4.6 \mathrm{pg} / \mathrm{mL}$ & 0.817 & 58.1 & 100 \\
CRP $^{15}$ & $3.5 \mathrm{mg} / \mathrm{L}$ & 0.906 & 82 & 88 \\
Pantraxin $^{16}$ & $2.45 \mathrm{ng} / \mathrm{mL}$ & 0.85 & 70.6 & 94.3 \\
Ferritin $^{17}$ & $196 \mathrm{ng} / \mathrm{mL}$ & 0.73 & 64.2 & 76.5 \\
SPEA $^{18}$ & $1,134 \mathrm{U} / \mathrm{L}$ & 0.85 & 84 & 82 \\
SRAGE $^{19}$ & $1,309 \mathrm{pg} / \mathrm{mL}$ & 0.77 & 75 & 71.4 \\
\hline
\end{tabular}

TNF- $\alpha$, Tumor necrosis factor - $\alpha$; IL-6, Interleukin-6, CRP, C-reactive protein; SPEA, Serum prolidase enzyme activity; sRAGE, Souble receptor for advanced glycation end product.

Table 2. Panel test for predicting non-alcoholic steatohepatitis

\begin{tabular}{lcccc}
\hline & AUROC & Sensitivity & Specificity & \multicolumn{1}{c}{ Validation } \\
\hline Nash Test $^{22}$ & 0.79 & $33 \%$ & $94 \%$ & Sensitivity was 21\% in validation study \\
NASH diagnostics $^{23}$ & 0.85 & $72 \%$ & $91 \%$ & AUROC was 0.7 in validation study \\
NASH model of NAFLD diagnostic panel $^{24}$ & 0.81 & $91 \%$ & $47 \%$ & \\
Nice model $^{25}$ & $0.83-0.88$ & $84 \%$ & $86 \%$ & \\
HAIR $^{26}$ & 0.90 & $80 \%$ & $89 \%$ & \\
\hline
\end{tabular}

NASH, non-alcoholic steatohepatitis; NAFLD, non-alcoholic fatty liver disease; AUROC, areas under the receiver-operating curve; HAIR, hypertension; ALT, insulin resistance.

Table 3. Panel test for predicting non-alcoholic steatohepatitis

\begin{tabular}{lccc}
\hline & Components & Sensitivity & Specificity \\
\hline NAFLD fibrosis score & & 0.77 & 0.96 \\
APRI $^{28}$ & Age, hyperglycemia, BMl, platelets, albumin, AST/ALT ratio & 0.65 & 0.97 \\
ELF $^{29}$ & AST, platelets & 0.80 & 0.90 \\
FibroTest $^{30}$ & Hyaluronic acid, TIMP1, PIIINP & 0.88 & 0.99 \\
BARD $^{31}$ & Total bilirubin, GGT, a2-macroglobulin, ApoA1, haptoglobin & 0.62 & 0.66 \\
\hline
\end{tabular}

NAFLD, non-alcoholic fatty liver disease; BMI, body mass index; AST, aspartate aminotransferase; ALT, alanine aminotransferase; APRI, AST-to-platelet ratio index; ELF, enhanced liver fibrosis panel; TIMP1, tissue inhibitor of matrix metalloproteinase 1; PIIINP, procollagen III amino-terminal peptide; GGT, $\gamma$-glutamyltransferase; ApoA1: apolipoprotein A1.

cantly increased compared with normal or simple steatosis. CK-18 showed relatively good results (sensitivity $78 \%$, specificity $87 \%$, areas under the receiver-operating curve (AUROC) 0.82) in some precedent studies, demonstrating the potential for screening nonalcoholic steatohepatitis. However, with CK-18 in clinical application demonstrates a large variation in cut-off values compared to previous studies, and its sensitivity and specificity to predict steatohepatitis was not satisfactory. ${ }^{20}$ A meta-analysis using 11 recent studies shows that AUROC for NASH ranged from 0.71-0.93, sensitivity was $66 \%$, and specificity was $82 \%$. Optimal cut-off values may increase sensitivity to $82 \%$ and specificity to $98 \%$, but there is a wide variation in cut-off values reported by each researcher (optimal cut off $136-338 \mathrm{U} / \mathrm{L}$ ). ${ }^{21}$ For this reason, the
American Association for the Study of Liver Diseases (AASLD) as well as practitioners in Korea have not recommended the use of CK-18 for diagnosis and treatment of NASH.

The Nash Test, ${ }^{22}$ NASH diagnostics, ${ }^{23}$ NASH model of NAFLD diagnostic panel, ${ }^{24}$ Nice model, ${ }^{25}$ and HAIR score ${ }^{26}$ have been suggested as biochemical tests to predict NASH, but in most studies, sensitivity was low and AUROC values were unsatisfactory (Table 2). However, the biggest drawback is that most models have not been validated externally, indicating that further studies are needed for confirmation. For instance, HAIR has a relatively high AUROC, sensitivity, and specificity, but also has the limitation of efficacy only in the severely obese patient, with no external validation for other NASH patients. ${ }^{26}$ 


\section{Advanced fibrosis $\geq \mathrm{F} 3$}

NAFLD fibrosis score, ${ }^{27}$ APRI (AST-to-platelet ratio index), ${ }^{28}$ ELF (enhanced liver fibrosis panel), ${ }^{29}$ FibroTest ${ }^{30}$ and BARD $^{31}$ have been studied to predict advanced fibrosis $\geq F$ 3 (Table 3 ) in NAFLD patients. ELF (Hyaluronic acid, TIMP1, PIIINP) and FibroTest ( $\alpha 2-$ macroglobulin, ApoA1, haptoglobin) use direct markers from collagen synthesis and degradation, while the NAFLD fibrosis score and APRI exploit biochemical test components, such as age, glucose, body mass index, platelets and albumin, which are commonly used in clinical practice.

The NAFLD Fibrosis score has been studied extensively studied worldwide. It is composed of six markers (age, hyperglycemia, $\mathrm{BMI}$, platelet, albumin, and AST/ALT ratio), which are clinically or biochemically measured easily. The NAFLD fibrosis score typically uses low and high cut-off values. When a low cut-off value (-1.455) was used, a negative predictive value (NPV) for predicting without advanced fibrosis (<F3) was 93\%, and when a high cut-off value (0.676) was used, the positive predictive value (PPV) for predicting advanced fibrosis ( $\geq$ F3) was $90 \%{ }^{27}$ Since then, the NAFLD fibrosis score has been validated externally in Western countries, demonstrating relatively reliable predictability compared to other models for prediction of fibrosis. However, the results of studies on external validation using the NAFLD fibrosis score conducted in Eastern countries showed somewhat different results. One study conducted on 162 NAFLD patients in Hong Kong indicated NPVs of low cut-off value were very high at 91\%, while PPVs of high cut-off value were $0 \%{ }^{32}$ In a Hong Kong cohort of obese subjects (average BMI 28.5), only 18 patients (11\%) had advanced fibrosis $\geq \mathrm{F} 3^{32}$ In a Japanese multicenter study conducted on 588 subjects showed the NPV of low cut-off was $98 \%$, while the PPV of high cut-off was $43 \%{ }^{33}$ Advanced fibrosis $\geq \mathrm{F} 3$ was detected in $27.8 \%$ of these subjects. ${ }^{33}$ In a study on external validation of the NAFLD fibrosis score using a Korean cohort, AUROC to predict advanced fibrosis was high at 0.964 . The NPV of low cut-off was $100 \%$, while the PPV of high cut-off was 33.3\%. ${ }^{34}$ Therefore, the use of PPV with a high cut-off in Asian countries in patients with low advanced fibrosis needs further study.

\section{DIAGNOSIS OF NAFLD: AN INVASIVE METHODS (liver biopsy)}

Currently, liver biopsy is the gold standard for diagnosis of NAFLD. However, this method still has several unresolved issues.
Occasionally, advanced NASH-associated cirrhosis may not demonstrate fat in the liver, and is called "burned-out cirrhosis." This condition can't be easily distinguished from cryptogenic cirrhosis. Perisinusoidal fibrosis, ballooning hepatocytes, and Mallory-Denk bodies found in non-alcoholic patients may be a clue for NASHassociated cirrhosis, but it is difficult to distinguish from "burnedout" cirrhosis unless confirmed by biopsy. Also, liver biopsy utilizes only a small section of the liver $\left(1 / 50,000^{\text {th }}\right)$, and different positions may lead to different results. In regard to steatosis, the extent of fat accumulation varies depending upon in which segment it is located, as the amount of hepatic fat is significantly higher in the right lobe than the left. ${ }^{35}$ There are significant differences in the amount of intrahepatic fat between the various lobes and segments. ${ }^{35}$ In addition, needle biopsy and surgical biopsy can result in different findings for the same organ, as anesthesia during a surgical biopsy may cause minor or "surgical" hepatitis. ${ }^{36}$ In many cases, liver tissue can be obtained from the surface of the liver rather than its central core. Surface tissue often presents an expanded portal vein and exaggerated fibrosis. Also, observer-toobserver agreement may be low in the interpretation the liver biopsy. Finally, though several diagnostic criteria for NASH are recommended, there are no unified standards at the present time. Currently, three standards are being used for histological diagnosis of NASH in clinical practice, the Brunt system, NASH CRN, and the SAF/FLIP algorithm. The Brunt system is the prototype of histological diagnostic criteria for nonalcoholic fatty liver diseases. ${ }^{37}$ This system divides steatosis, hepatocyte ballooning degeneration, lobular and portal vein inflammation into three types; mild, moderate and severe and classifies liver fibrosis into 4 stages. $^{37}$ It stresses hepatocyte ballooning, fatty accumulation in the liver, and inflammatory cell infiltration, especially on damage in zone 3. However, this system has not been corroborated extensively by other researchers. NAS is recommended by the non-alcoholic steatohepatitis Clinical Research Network (CRN), and its clinical usefulness has been validated by a number of researchers. ${ }^{38}$ NAS was not designed to diagnose NASH. but it was designed to assess treatment efficacy in a clinical study on fatty liver diseases. The NAS system contains components that determine fibrosis, but the NAS score itself does not include a determination of fibrosis. For this reason, there are cases with advanced hepatic fibrosis and low NAS scores or the exact opposite. Twenty-eight percent of obvious NASH patients scored less than 5 points in the study, while $7 \%$ of obvious non-NASH patients scored more than 5 points. ${ }^{39}$ Recently, the European Fatty Liver Inhibition of Progression (FLIP) group has recommended the SAF score. ${ }^{40,41}$ SAF, a 
scoring system for Steatosis, Activity (inflammation), and Fibrosis, puts weight on hepatic fibrosis and hepatocyte ballooning to diagnose NASH, unlike the NAS score. SAF also has the advantage of less difference in interpretation by multiple observers. Worldwide consensus on diagnostic criteria and severity in NASH should be developed.

In spite of the issues listed above, liver biopsy is still the gold standard in practice and clinical trial. Globally unified standards for histological diagnosis, methods to enhance agreement in interpretation, and standardization of biopsy sites and methods still require resolution.

\section{TREATMENT UPDATE ON NAFLD}

\section{Lifestyle modification}

The treatment of NAFLD is based on weight loss achieved by lifestyle modification. A minimum of $5 \%$ weight loss decreases hepatic fat, and a 7-10\% weight loss is required to improve hepatic inflammation. ${ }^{42,43}$ However, it is difficult for diet and exercise regimens to achieve and maintain a $10 \%$ weight loss. In a recent study conducted in Cuba, a low calorie diet and modification of habits achieved a $30 \%$ weight loss in subjects for one year. ${ }^{44}$ However, only $19 \%$ of the subjects achieved improvement in hepatic fibrosis. Instead, $16 \%$ of them had advanced hepatic fibrosis. Though modification of habit and diet is the foundation for the treatment of nonalcoholic fatty liver disease, there still exists a lack of randomized controlled trials on diet, modification of habit, and weight loss on the improvement of NASH and hepatic fibrosis. In the past, aerobic exercise was considered a better way to reduce hepatic fat than weight training, but recent studies show that a combination of aerobic exercise and weight training is more effective than an aerobic exercise alone.

\section{Pharmacotherapy}

In some randomized controlled trials, the effect of vitamin $\mathrm{E}$ and obeticholic acid on nonalcoholic fatty liver diseases has been proven, ${ }^{45,46}$ but these drugs have not been approved in most countries. The following drugs are used off-label for NAFLD in Korea.

FXR agonist (Obeticholic acid, Intercept, Phase Ilb): Farnesoid X Receptor (FXR) agonist (obeticholic acid) has significantly decreased hepatic inflammation in large scale clinical trial in patients with NASH. ${ }^{46}$ However, at the same time obeticholic acid has significantly increased blood triglyceride and LDL-cholesterol levels and decreased HDL-cholesterol concentration. Given that many NASH patients die due to cardiovascular diseases, an increased blood cholesterol level due to the use of obeticholic acid is problematic. ${ }^{47}$ Recently, intestine-specific FXR agonist has been investigated. This new FXR agonist has reduced inflammatory cell infiltration around fat cells and improved insulin resistance, with a decline in hepatic fat and inflammation in preclinical studies. In addition, the intestine-specific FXR agonist did not increase cholesterol and triglyceride concentration. ${ }^{48}$ Further studies on human subjects are warranted.

NOX-1/4 Inhibitor (GKT137831, Genkyotex): GKT137831 is a first-in-class drug targeting NADPH oxidase (NOX) 1 and 4. GKT137831 was designed as an anti-diabetic drug. The NOX1/4 inhibitor failed to reduce albuminuria in patients with diabetic nephropathy, but it showed an excellent safety profile. GKT137831 extended its therapeutic targets, including atherosclerosis, idiopathic pulmonary fibrosis, liver fibrosis, and models of angiogenesis. NOX embedded in inflammatory and hepatic stellate cells plays a crucial role in the outbreak of hepatic inflammation and fibrosis. The overexpression of NOX 1 and 4 in tissue from patients with liver cirrhosis and NASH confirms the accuracy of the preclinical models. The NOX1/4 inhibitor has had a favorable effect on the inhibition of hepatic inflammation and fibrosis in animal testing. ${ }^{49}$ Another interesting finding in preclinical data is that the NOX1/4 inhibitor decreases hepatocyte apoptosis.

Galectin-3 inhibitor (GR-MD-02, Galectin Therapeutics, Phase II): Galectin-3, a kind of carbohydrate-binding protein, is bound to the galactose residues of large proteins such as glycoprotein. Galectin-3 normally expresses in immune cells at a very low concentration and increases inflammation. ${ }^{50}$ Galectin-3 antagonist has demonstrated an outstanding result in animal study. Galectin-3 knock-out mice have had lesser hepatic fibrosis. GRMD-02 (Galectin Therapeutics, Inc, Norcross, GA, USA) has no obviously identified mechanism, but acts as an antagonist on galectin. In a phase I study on 31 subjects with biopsy-proven NASH with advanced fibrosis (Brunt stage 3), a high dose of GR-MD-02 $(8 \mathrm{mg} / \mathrm{kg})$ administered for 6 weeks was relatively safe and lowered FibroTest ${ }^{\circledR}$ and $\alpha 2$-macroglobulin levels. At this time, GRMD-02 is undergoing Phase II clinical trials for safety and efficacy (ClinicalTrial.gov NCT02462967).

CCR2 and CCR5 inhibitor (Cenicriviroc, Tobira, Phase IIb): C-C chemokine receptor (CCR) is expressed in diverse immune cells, including monocytes, macrophagocytes, and hepatic Kupffer cells. Cenicriviroc ${ }^{\circledR}$ was developed as anti-viral agent in 
HIV-1 patients. It has shown effective antiviral activity and safety in recent phase Ilb clinical trials in patient with AIDS. Cenicriviroc ${ }^{\circledR}$ also activated hepatic stellate cells and hepatic fibrosis. ${ }^{51}$ Cenicriviroc ${ }^{\circledR}$ has induced histological improvement in both models of diet-inducing steatosis and hepatic fibrosis in animals. Currently, clinical studies on NASH patients with CENTAUR using Cenicriviroc $^{\circledR}$ are in progress, ${ }^{51}$ awaiting the results of Cenicriviroc ${ }^{\circledR}$ safety and efficacy (ClinicalTrial.gov NCT02217475).

Pan-caspase inhibitor (Emricasan, Conatus Pharmaceuticals, Inc., Phase Ila): This anti-apoptotic drug has already received attention as a potential target for NASH, because hepatocyte apoptosis is the most important step in developing NASH. For this reason, hepatocyte ballooning, which is surrogate marker of hepatocyte apoptosis, is important in the diagnosis of liver pathology. Emricasan ${ }^{\circledR}$ suppressed liver injury and fibrosis by inhibiting hepatocytes apoptosis. ${ }^{52}$ In a phase II clinical study on 38 NASH patients, Emricasan ${ }^{\circledR}$ lowered serum aminotransferase activity and cleaved CK-8 serum concentration..$^{53}$ Emricasan ${ }^{\circledR}$ has been studied across a broad range of liver disease etiologies and stages of progression, and has demonstrated meaningful reductions in hepatic venous pressure gradient (HVPG) in cirrhotic patients. ENCORE trials designed to evaluate fibrosis and inflammation in patients with NASH fibrosis showed improvement in HVPG in NASH cirrhosis in November of 2015. Recently the U.S. FDA has granted Fast Track designation to the company's Emricasan ${ }^{\circledR}$ development program for the treatment of cirrhosis caused by nonalcoholic steatohepatitis.

PPAR- $\alpha / \delta$ agonist (GFT505, Genfit, Phase Ilb): This agent was developed as a PPAR- $\alpha / \delta$ agonist, and is already known for mitigation of hepatic fat and relief of inflammation. Very little is known of the role of PPAR- $\delta$, but it has the effect of improving the function of mitochondria, fat burning, and insulin resistance. In a large-scale randomized controlled trial on 274 NASH patients, GFT505 did not significantly decrease hepatic inflammation. ${ }^{54}$ However, when administered to a subgroup with the NAS score $\geq 4$ before treatment, GFT505 induced significant improvement in hepatic inflammation. Further studies on target groups and timing of optimal clinical application of GFT505 are in progress.

SCD-1 inhibitor (Aramchol, Galmed, Phase IIb): StearoylCoA desaturase-1 (SCD-1), an enzyme essential to neutral lipid synthesis, has a strong effect on controlling neutral lipid biosynthesis. In a study conducted for 3 months on 60 patients with NASH confirmed by liver biopsy, Aramchol ${ }^{\circledR}$ showed a significant decrease in hepatic fat of roughly $12.6 \% .{ }^{6}$ However, only 6 (10\%) of the above subjects were NASH patients, and the anti-fibrotic effect was not evaluated. Currently, a phase Ilb clinical study of 240 NASH patients treated with Aramchol ${ }^{\mathbb{B}}$ is in progress, and is attempting to determine the level of hepatic fibrosis and hepatic inflammation in a non-invasive way (ClincialTrails.gov NCT02279524).

Apoptosis signal-regulating kinase 1 inhibitor (GS-4997, Gilead, Phase II): Apoptosis signal-regulating kinase 1 (ASK1) is activated by various stimuli, including hyperglycemia, TGF- $\beta$, and oxidative stimulus in the body. Activated ASK1 induces apoptosis and fibrosis through p38 and JNK1. In the animal NASH model, the ASK1 antagonist induced a decrease in hepatic fat and fibrosis. It also induced improvement in insulin resistance and metabolic index by prompting weight loss. ${ }^{55}$ Presently, a phase II clinical study on the ASK1 antagonist (GS-4997) is in progress testing NASH patients with moderate steatosis and advanced fibrosis (ClinicalTrial.gov NCT02466516).

Lysyk oxidase-like 2 inhibitor (Simtuzumab, Gilead, Phase Ilb): This drug strengthens collagen binding in the extracellular matrix to restrain decomposition. In the hepatic cirrhosis model, more LOXL2 appeared. In the animal model, the LOXL2 monoclonal antibody restrained hepatic fibrosis. ${ }^{56} \mathrm{~A}$ phase $\mathrm{llb}$ study on the inhibition of hepatic fibrosis and cirrhosis in nonalcoholic fatty liver disease treated with Simtuzumab (Gilead Sciences, Foster City, USA) is in progress. This study, targeting 222 NASH patients, follows patients for 6 years to assess the incidence of hepatic cirrhosis (ClincialTrial.gov NCT01672866). At the same time, a phase IIb clinical study on the safety and efficacy of simtuzumab for steatosis associated hepatic cirrhosis patients is in progress (ClincalTrial. gov NCT01672879). This study will target patients with compensated liver cirrhosis, assess the incidence of associated complications of hepatic cirrhosis. Simtuzumab will be studied with a reduced amount of HVPG after treatment for a maximum of 6 years with subsequent HVPG measurement. The study is expected to be completed in 2024.

\section{Conflicts of Interest}

The authors have no conflicts to disclose.

\section{REFERENCES}

1. Jeong EH, Jun DW, Cho YK, Choe YG, Ryu S, Lee SM, et al. Regional prevalence of non-alcoholic fatty liver disease in Seoul and Gyeonggi-do, Korea. Clin Mol Hepatol 2013;19:266-272.

2. Bohte $A E$, van Werven JR, Bipat S, Stoker J. The diagnostic accuracy of US, CT, MRI and 1H-MRS for the evaluation of hepatic steatosis com- 
pared with liver biopsy: a meta-analysis. Eur Radiol 2011;21:87-97.

3. Pickhardt PJ, Park SH, Hahn L, Lee SG, Bae KT, Yu ES. Specificity of unenhanced CT for non-invasive diagnosis of hepatic steatosis: implications for the investigation of the natural history of incidental steatosis. Eur Radiol 2012;22:1075-1082.

4. Kang BK, Yu ES, Lee SS, Lee Y, Kim N, Sirlin CB, et al. Hepatic fat quantification: a prospective comparison of magnetic resonance spectroscopy and analysis methods for chemical-shift gradient echo magnetic resonance imaging with histologic assessment as the reference standard. Invest Radiol 2012;47:368-375.

5. Henninger B, Zoller $H$, Rauch S, Schocke M, Kannengiesser S, Zhong $X$, et al. Automated two-point dixon screening for the evaluation of hepatic steatosis and siderosis: comparison with R2-relaxometry and chemical shift-based sequences. Eur Radiol 2015;25:1356-1365.

6. Hu HH, Bornert P, Hernando D, Kellman P, Ma J, Reeder S, et al. ISMRM workshop on fat-water separation: insights, applications and progress in MRI. Magn Reson Med 2012;68:378-388.

7. Noureddin M, Lam J, Peterson MR, Middleton M, Hamilton G, Le $T A$, et al. Utility of magnetic resonance imaging versus histology for quantifying changes in liver fat in nonalcoholic fatty liver disease trials. Hepatology 2013;58:1930-1940.

8. Loomba R, Sirlin CB, Ang B, Bettencourt R, Jain R, Salotti J, et al. Ezetimibe for the treatment of nonalcoholic steatohepatitis: assessment by novel magnetic resonance imaging and magnetic resonance elastography in a randomized trial (MOZART trial). Hepatology 2015;61:1239-1250.

9. Tang A, Desai A, Hamilton G, Wolfson T, Gamst A, Lam J, et al. ACcuracy of MR imaging-estimated proton density fat fraction for classification of dichotomized histologic steatosis grades in nonalcoholic fatty liver disease. Radiology 2015;274:416-425.

10. Tang A, Tan J, Sun M, Hamilton G, Bydder M, Wolfson T, et al. Nonalcoholic fatty liver disease: MR imaging of liver proton density fat fraction to assess hepatic steatosis. Radiology 2013;267:422-431.

11. Bedogni G, Bellentani S, Miglioli L, Masutti F, Passalacqua M, Castiglione A, et al. The Fatty Liver Index: a simple and accurate predictor of hepatic steatosis in the general population. BMC Gastroenterol 2006;6:33.

12. Poynard T, Ratziu V, Naveau S, Thabut D, Charlotte F, Messous D, et al. The diagnostic value of biomarkers (SteatoTest) for the prediction of liver steatosis. Comp Hepatol 2005;4:10.

13. Alaaeddine N, Sidaoui J, Hilal G, Serhal R, Abedelrahman A, Khoury $S$. TNF-alpha messenger ribonucleic acid (mRNA) in patients with nonalcoholic steatohepatitis. Eur Cytokine Netw 2012;23:107-111.

14. Tarantino G, Conca P, Pasanisi F, Ariello M, Mastrolia M, Arena A, et al. Could inflammatory markers help diagnose nonalcoholic steatohepatitis? Eur J Gastroenterol Hepatol 2009;21:504-511.

15. Fierbinteanu-Braticevici C, Baicus C, Tribus L, Papacocea R. Predictive factors for nonalcoholic steatohepatitis (NASH) in patients with nonalcoholic fatty liver disease (NAFLD). J Gastrointestin Liver Dis 2011;20:153-159

16. Yoneda M, Uchiyama T, Kato S, Endo H, Fujita K, Yoneda K, et al. Plasma Pentraxin3 is a novel marker for nonalcoholic steatohepatitis (NASH). BMC Gastroenterol 2008;8:53.

17. Yoneda M, Nozaki Y, Endo H, Mawatari H, lida H, Fujita K, et al. Serum ferritin is a clinical biomarker in Japanese patients with nonalcoholic steatohepatitis (NASH) independent of HFE gene mutation. Dig Dis Sci 2010;55:808-814.

18. Tamimi TI, Elgouhari HM, Alkhouri N, Yerian LM, Berk MP, Lopez R, et al. An apoptosis panel for nonalcoholic steatohepatitis diagnosis. J Hepatol 2011;54:1224-1229.

19. Kayadibi H, Gültepe M, Yasar B, Ince AT, Ozcan O, Ipcioglu OM, et al. Diagnostic value of serum prolidase enzyme activity to predict the liver histological lesions in non-alcoholic fatty liver disease: a surrogate marker to distinguish steatohepatitis from simple steatosis. Dig Dis Sci 2009;54:1764-1771.

20. Cusi K, Chang Z, Harrison S, Lomonaco R, Bril F, Orsak B, et al. Limited value of plasma cytokeratin-18 as a biomarker for NASH and fibrosis in patients with non-alcoholic fatty liver disease. J Hepatol 2014;60:167-174.

21. Kwok R, Tse YK, Wong GL, Ha Y, Lee AU, Ngu MC, et al. Systematic review with meta-analysis: non-invasive assessment of non-alcoholic fatty liver disease--the role of transient elastography and plasma cytokeratin-18 fragments. Aliment Pharmacol Ther 2014;39:254269.

22. Poynard T, Ratziu V, Charlotte F, Messous D, Munteanu M, ImbertBismut $F$, et al. Diagnostic value of biochemical markers (NashTest) for the prediction of non alcoholo steato hepatitis in patients with non-alcoholic fatty liver disease. BMC Gastroenterol 2006;6:34.

23. Younossi ZM, Jarrar M, Nugent C, Randhawa M, Afendy M, Stepanova $M$, et al. A novel diagnostic biomarker panel for obesity-related nonalcoholic steatohepatitis (NASH). Obes Surg 2008;18:14301437.

24. Younossi ZM, Page S, Rafiq N, Birerdinc A, Stepanova M, Hossain $\mathrm{N}$, et al. A biomarker panel for non-alcoholic steatohepatitis (NASH) and NASH-related fibrosis. Obes Surg 2011;21:431-439.

25. Anty R, lannelli A, Patouraux S, Bonnafous S, Lavallard VJ, SenniBuratti $M$, et al. A new composite model including metabolic syndrome, alanine aminotransferase and cytokeratin-18 for the diagnosis of non-alcoholic steatohepatitis in morbidly obese patients. Aliment Pharmacol Ther 2010;32:1315-1322.

26. Dixon JB, Bhathal PS, O'Brien PE. Nonalcoholic fatty liver disease: predictors of nonalcoholic steatohepatitis and liver fibrosis in the severely obese. Gastroenterology 2001;121:91-100.

27. Angulo P, Hui JM, Marchesini G, Bugianesi E, George J, Farrell GC, et al. The NAFLD fibrosis score: a noninvasive system that identifies liver fibrosis in patients with NAFLD. Hepatology 2007;45:846-854. 
28. Wai CT, Greenson JK, Fontana RJ, Kalbfleisch JD, Marrero JA, Conjeevaram $\mathrm{HS}$, et al. A simple noninvasive index can predict both significant fibrosis and cirrhosis in patients with chronic hepatitis $C$. Hepatology 2003;38:518-526.

29. Guha IN, Parkes J, Roderick P, Chattopadhyay D, Cross R, Harris $S$, et al. Noninvasive markers of fibrosis in nonalcoholic fatty liver disease: Validating the European Liver Fibrosis Panel and exploring simple markers. Hepatology 2008;47:455-460.

30. Ratziu V, Massard J, Charlotte F, Messous D, Imbert-Bismut F, Bonyhay $L$, et al. Diagnostic value of biochemical markers (FibroTestFibroSURE) for the prediction of liver fibrosis in patients with nonalcoholic fatty liver disease. BMC Gastroenterol 2006;6:6.

31. Harrison SA, Oliver D, Arnold HL, Gogia S, Neuschwander-Tetri $B A$. Development and validation of a simple NAFLD clinical scoring system for identifying patients without advanced disease. Gut 2008;57:1441-1447.

32. Wong VW, Wong GL, Chim AM, Tse AM, Tsang SW, Hui AY, et al. Validation of the NAFLD fibrosis score in a Chinese population with low prevalence of advanced fibrosis. Am J Gastroenterol 2008;103:1682-1688.

33. Sumida $Y$, Yoneda $M$, Hyogo $H$, Yamaguchi $K$, Ono $M$, Fujii $H$, et al. A simple clinical scoring system using ferritin, fasting insulin, and type IV collagen 75 for predicting steatohepatitis in nonalcoholic fatty liver disease. J Gastroenterol 2011;46:257-268.

34. Yu SJ, Kim DH, Lee JH, Chung GE, Yim JY, Park MJ, et al. Validation of P2/MS and other noninvasive fibrosis scoring systems in the Korean population with nonalcoholic fatty liver disease. Korean J Gastroenterol 2011;57:19-27.

35. Bonekamp S, Tang A, Mashhood A, Wolfson T, Changchien C, Middleton MS, et al. Spatial distribution of MRI-Determined hepatic proton density fat fraction in adults with nonalcoholic fatty liver disease. J Magn Reson Imaging 2014;39:1525-1532.

36. Brunt EM. Nonalcoholic fatty liver disease: pros and cons of histologic systems of evaluation. Int J Mol Sci 2016;17. Pii: E97.

37. Brunt EM, Janney CG, Di Bisceglie AM, Neuschwander-Tetri BA, Bacon BR. Nonalcoholic steatohepatitis: a proposal for grading and staging the histological lesions. Am J Gastroenterol 1999;94:24672474.

38. Kleiner DE, Brunt EM, Van Natta M, Behling C, Contos MJ, Cummings $\mathrm{OW}$, et al. Design and validation of a histological scoring system for nonalcoholic fatty liver disease. Hepatology 2005;41:13131321.

39. Brunt EM, Kleiner DE, Wilson LA, Belt $P$, Neuschwander-Tetri BA; NASH Clinical Research Network (CRN). Nonalcoholic fatty liver disease (NAFLD) activity score and the histopathologic diagnosis in NAFLD: distinct clinicopathologic meanings. Hepatology 2011;53:810-820.

40. Bedossa P; FLIP Pathology Consortium. Utility and appropriateness of the fatty liver inhibition of progression (FLIP) algorithm and steatosis, activity, and fibrosis (SAF) score in the evaluation of biopsies of nonalcoholic fatty liver disease. Hepatology 2014;60:565-575.

41. Bedossa P, Poitou C, Veyrie N, Bouillot JL, Basdevant A, Paradis V, et al. Histopathological algorithm and scoring system for evaluation of liver lesions in morbidly obese patients. Hepatology 2012;56:17511759.

42. Lazo M, Solga SF, Horska A, Bonekamp S, Diehl AM, Brancati $\mathrm{FL}$, et al. Effect of a 12-month intensive lifestyle intervention on hepatic steatosis in adults with type 2 diabetes. Diabetes Care 2010;33:2156-2163.

43. Schafer S, Kantartzis K, Machann J, Venter C, Niess A, Schick F, et al. Lifestyle intervention in individuals with normal versus impaired glucose tolerance. Eur J Clin Invest 2007;37:535-543.

44. Vilar-Gomez E, Martinez-Perez Y, Calzadilla-Bertot L, Torres-Gonzalez A, Gra-Oramas B, Gonzalez-Fabian L, et al. Weight Loss Through Lifestyle Modification Significantly Reduces Features of Nonalcoholic Steatohepatitis. Gastroenterology 2015;149:367-378.

45. Sanyal AJ, Chalasani N, Kowdley KV, McCullough A, Diehl AM, Bass $N M$, et al. Pioglitazone, vitamin $E$, or placebo for nonalcoholic steatohepatitis. N Engl J Med 2010;362:1675-1685.

46. Neuschwander-Tetri BA, Loomba R, Sanyal AJ, Lavine JE, Van Natta $\mathrm{ML}$, Abdelmalek MF, et al. Farnesoid $\mathrm{X}$ nuclear receptor ligand obeticholic acid for non-cirrhotic, non-alcoholic steatohepatitis (FLINT): a multicentre, randomised, placebo-controlled trial. Lancet 2015;385:956-965.

47. Lawlor DA, Callaway M, Macdonald-Wallis C, Anderson E, Fraser A, Howe LD, et al. Nonalcoholic fatty liver disease, liver fibrosis, and cardiometabolic risk factors in adolescence: a cross-sectional study of 1874 general population adolescents. J Clin Endocrinol Metab 2014;99:E410-E417.

48. Fang S, Suh JM, Reilly SM, Yu E, Osborn O, Lackey D, et al. Intestinal FXR agonism promotes adipose tissue browning and reduces obesity and insulin resistance. Nat Med 2015;21:159-165.

49. Aoyama T, Paik YH, Watanabe S, Laleu B, Gaggini F, Fioraso-Cartier $\mathrm{L}$, et al. Nicotinamide adenine dinucleotide phosphate oxidase in experimental liver fibrosis: GKT137831 as a novel potential therapeutic agent. Hepatology 2012;56:2316-2327.

50. Yang RY, Rabinovich GA, Liu FT. Galectins: structure, function and therapeutic potential. Expert Rev Mol Med 2008;10:e17.

51. Lefebvre E, Gottwald M, Lasseter K, Chang W, Willett M, Smith PF, et al. Pharmacokinetics, Safety, and CCR2/CCR5 Antagonist Activity of Cenicriviroc in Participants With Mild or Moderate Hepatic Impairment. Clin Transl Sci 2016:9:139-148.

52. Barreyro FJ, Holod S, Finocchietto PV, Camino AM, Aquino JB, Avagnina A, et al. The pan-caspase inhibitor Emricasan (IDN-6556) decreases liver injury and fibrosis in a murine model of non-alcoholic steatohepatitis. Liver Int 2015;35:953-966. 
53. Shiffman M, Freilich B, Vuppalanchi R, Watt K, Burgess G, Morris M, et al. A placebo-controlled, multicenter, double-blind, randomised trial of emricasan (IDN-6556) in subjects with non-alcoholic fatty liver disease (NAFLD) and raised transaminases. J Hepatol 2015;62(Suppl 2):S282.

54. Ratziu V, Harrison SA, Francque SM, Bedossa P, Serfaty L, RomeroGomez $\mathrm{M}$, et al. An international, phase 2 randomized controlled trial of the dual PPAR $\alpha-\delta$ agonist GFT505 in adult patients with
NASH. Hepatology 2015;62(Suppl 1): 262A-263A.

55. Xiang $M$, Wang PX, Wang AB, Zhang XJ, Zhang $Y$, Zhang $P$, et al. Targeting hepatic TRAF1-ASK1 signaling to improve inflammation, insulin resistance, and hepatic steatosis. J Hepatol 2016;64:1365-1377.

56. Barry-Hamilton V, Spangler R, Marshall D, McCauley S, Rodriguez HM, Oyasu M, et al. Allosteric inhibition of lysyl oxidase-like-2 impedes the development of a pathologic microenvironment. Nat Med 2010;16:1009-1017. 